\title{
Universidad, ciudadanía y TIC
}

\author{
José Ignacio "Iñaki” Chaves*
}

Recibido: 22 de agosto de 2014 - Aprobado: 16 de octubre de 2014

¿Está el mundo del siglo XXI del revés? Si miramos las noticias que nos venden los medios masivos de difusión de noticias y si analizamos la realidad social que nos rodea, la respuesta es SÍ. Si el mundo está patas arriba significa que la escuela, que forma parte de ese mundo, también se encuentra así. Dice Eduardo Galeano que si "(...) el plomo aprende a flotar y el corcho, a hundirse. Las víboras aprenden a volar y las nubes aprenden a arrastrarse por los caminos" es que el mundo definitivamente está al revés. ¿Por qué este planteamiento inicial para hablar de Universidad, de ciudadanía y de nuevas tecnologías? Porque considero que en la situación del mundo de hoy, digital y pro-tecnológico en lo comunicativo, depredador y ciego en lo medioambiental, neoliberal y dictatorial en lo político, y capitalista y explotador en lo económico, tienen un rol relevante los tres campos enunciados en el título del presente artículo.

Palabras clave: TIC, ciudadanía, Universidad, reeducación, participación social.

Sociólogo de la Universidad Complutense de Madrid. DEA en Sociología de la misma Universidad. Candidato a Doctor en Comunicación y Ciencias Sociales de la Universidad Rey Juan Carlos de Madrid. Actualmente se desempeña como profesor de la Maestría en Comunicación, Desarrollo y Cambio Social de la Universidad Santo Tomás. Contacto: josechaves@usantotomas.edu.co 


\title{
University, citizenship and ICT
}

\author{
José Ignacio "Iñaki" Chaves”
}

\section{A bstract}

Is the twenty first world upside down? If we see the news from Mass Media and we analyze our social reality, the answer is YES. If the world is upside down this means that academy, which is part of this world, is also in that way. Eduardo Galeano says "(...) lead learns how to float and cork to sink. Vipers learn how to fly and clouds learn how to crawl" this means that the world is upside down. Why this introductory statement in order to talk about university, citizenship and new technologies? Because I consider that in today world's situation, which is digital and pro technology in communicative areas, predator and blind in environmental issues, neoliberal and dictatorial in political fields, and capitalist and operator in economic areas, have a key role in the three main subjects of this article.

Keywords: ICT, citizenship, university, reeducation, social participation.

* Sociologist at Universidad Complutense of Madrid. Master in Sociology from this University. PhD candidate in Communication and Social Sciences from Universidad Rey Juan Carlos of Madrid. Currently, he is professor of the Master Program in Communication, Development and Social Change at Universidad Santo Tomás. Address: Carrera 9, 51-11, Bogotá, Colombia. Email: josechaves@usantotomas.edu.co. 


\title{
L'université, la citoyenneté et TIC
}

\author{
José Ignacio “Iñaki” Chaves*
}

\section{Résumé}

Est-il le monde du XXIe siècle au revers? Si on regarde les nouvelles qu'on vendent les médias massifs de diffusion et si on analyse la réalité sociale qui nous entoure, la réponse est affirmative. Si le monde est bouleversé signifie que l'école qui fait partie de ce monde, elle est comme ça. Eduardo Galeano dit que si «le plomb apprend à flotter et le liège à s'écrouler. Les vipères apprennent à voler et les nuages apprennent à ramper par les chemins» c'est que le monde est certainement à l'envers. Pourquoi cette approche initial pour parler de l'université, de citoyenneté et de nouvelles technologies? Parce que je considère que dans la situation du monde d'aujourd'hui, digital et pro-technologique dans le communicatif, prédateur et aveugle dans l'environnemental, néolibéral et dictatorial dans le politique, et capitaliste et exploiteur dans l'économique, les trois champs énoncés dans le titre de l'article présent ont un rôle éminent.

Mots clés: TIC, la citoyenneté, l' université, la rééducation, la participation sociale.

Sociologue de l'Université Complutense de Madrid. DEA en Sociologie de la même Université. Candidat à Docteur en Communication et Sciences Sociales de l'Université Rey Juan Carlos de Madrid. Actuellement il est professeur de la Maîtrise en Communication, Développement et changement social à l'Université SantoTomas. Adresse: Carrera 9, 51-11, Bogotá, Colombia. Email: josechaves@usantotomas.edu.co. 
¡Estudia lo elemental! Para aquellos

cuya hora ha llegado

no es nunca demasiado tarde.

¡Estudia el «abc»! No basta, pero

estúdialo, ¡No te canses!

¡Empieza! ¡Tú tienes que saberlo todo!

¡Estudia, hombre en el asilo!

¡Estudia, hombre en la cárcel!

¡Estudia, mujer en la cocina!

¡Estudia, sexagenario!

¡Asiste a la escuela, desamparado!

¡Persigue el saber, muerto de frío!

¡Empuña el libro, hambriento! ¡Es un arma!

¡No temas preguntar, compañero!

¡No te dejes convencer!

¡Compruébalo tú mismo!

Lo que no sabes por ti,

no lo sabes.

(Brecht, 1999, p. 30).

Esto nos decía Bertolt Brecht en 1933. En ese poema está implícita la Universidad, a través del estudio, y está presente la ciudadanía en esos hombres y mujeres encerrados, muertos de hambre o desamparados. No están las TIC porque no habían llegado, pero sí está presente una poderosa herramienta que, de otra manera, usamos hoy a través de las nuevas tecnologías: el libro.

La educación, incluida la universitaria, tiene un papel muy destacado en la construcción de espacios de debate democrático para la ciudadanía. Las TIC también pueden contribuir a ello.

\section{Protagonistas}

Ciudadanía: podemos decir que es el conjunto de prácticas que hacen que una persona sea integrante del pleno derecho de una sociedad cualquiera. La ciudadanía formal es la que supone poseer un documento identificativo, la sustantiva es la que define los derechos y obligaciones que tiene la persona en una comunidad política. Esos derechos y esas obligaciones exigen que las instituciones públicas respondan sobre los compromisos de participación. 
Universidad: es la institución de enseñanza superior donde la ciudadanía se forma en diversas materias y recibe un título por el grado académico alcanzado. En ella también se investiga y se produce conocimiento científico.

TIC: las tecnologías de la información y la comunicación serían todos aquellos elementos que se utilizan en la emisión, procesamiento y recepción de datos e información. Se habla de NTIC, nuevas tecnologías, pero en todas las épocas ha habido alguna tecnología que fue nueva y hoy puede ser hasta obsoleta.

La ciudadanía se forma en la escuela, además de en la familia y en la calle, y la Universidad es una parte importante, y además superior, de esa escuela; la conformación y el reconocimiento de la ciudadanía es, probablemente, como dice el profesor Giner, el mayor logro de la humanidad (Giner, s.f., p. 1), y las TIC son usadas por la ciudadanía, muchas veces de manera "impuesta" por el sistema, y son utilizadas por la Universidad en sus procesos formativos. La ciudadanía, cierto número de sus miembros, pasa por la Universidad, y hace uso, o debería hacerlo, de las herramientas tecnológicas para reivindicar su papel y su lugar en la sociedad.

Los tres sujetos del presente ensayo conforman este mundo al revés, los tres están interrelacionados y los tres son protagonistas y tienen una parte de responsabilidad en el estado actual del planeta.

¿En qué situación se encuentran la escuela, la academia, la Universidad en este siglo XXI? ¿Qué educación y qué valores transmitimos desde las instituciones educativas? ¿Qué papel juegan las TIC en esta tarea?

Existe un grave problema hoy, y es que las universidades están enseñando en sus aulas ese mundo al revés, haciendo uso o sin hacerlo de esas tecnologías, e ignorando muchas veces que sus estudiantes tienen que ser formados como ciudadanas y ciudadanos antes que como expertos en esta o aquella materia, en esa o aquella rama del saber. Porque la ciudadanía es una construcción social que se mejora aprendiéndola y ejerciéndola. La Universidad y las TIC tienen que servir para esa obra.

Las preguntas planteadas un poco más arriba no son de fácil respuesta. Y la teoría y sus teóricos nos presentan variadas y diferentes contestaciones en función de intereses políticos o ideológicos.

\section{La Universidad, centro del debate}

La institución universitaria es el centro de este ensayo porque en ella se pueden conjugar los otros dos actores que la acompañan: la ciudadanía y las TIC.

Sobre la Universidad se ha dicho mucho. Por ejemplo, Derrida se preguntaba: 
¿Cómo no hablar, hoy, de la Universidad? (...) No se puede no hablar de ella. (...) ¿Existe hoy en día, en lo que respecta a la Universidad, lo que se llama una "razón de ser"? (...) la esencia de la Universidad en su relación con la razón y con el ser, pero también la causa, la finalidad, la necesidad, las justificaciones, el sentido, la misión, en una palabra, la destinación de la Universidad. Tener una "razón de ser" es tener una justificación para existir, tener un sentido, una finalidad, una destinación (Derrida, 1997, p. 1).

Y el profesor Martín Barbero propone, y así lo expresó en la conferencia que dictó para inaugurar el segundo semestre de 2013 de la Facultad de Comunicación en la Universidad Santo Tomás, que se debe "meter" más país en la Universidad. Es un planteamiento que parte de una constatación: la Universidad hoy vive algo alejada de la realidad social que la rodea. Está más pendiente del mercado que de la ciudadanía.

La presidenta de la Asociación de Profesores de la Universidad de Antioquia, dice en la presentación del texto de Gutiérrez-Girardot (2011, p. 8), que la Universidad es "una institución que nunca pasará de moda como objeto de reflexión, dada su importancia en la sociedad y en la construcción y/o consolidación de la democracia".

No me atrevería yo a decir con tanta seguridad que nunca pasará de moda, además no creo que sea la Universidad una institución que tenga que gastar esfuerzos en peleas por mantenerse de moda. Su campo de batalla deber ser otro.

Hoy, es necesario hablar de la Universidad, de la escuela, de la educación, de la enseñanza, desde un punto de vista crítico sin temer poner sobre la mesa sus debilidades, que las tiene, y sin olvidar todas sus fortalezas, que son muchas. También discutir sobre la ciudadanía, para que defienda su lugar y recupere, si es que alguna vez lo tuvo, su papel en la sociedad, a la que constituye y de la que forma parte. Y cómo no, de las TIC, porque si la Universidad se tecnifica en exceso, si se deja seducir por el aura tecnológica y descuida el intercambio, la interacción y el contacto, caerá en la pérdida de uno de sus mayores valores: la comunicación educativa, o educomunicación, algo que, como decían Freire y Kaplún et al. no se da en el aula virtual sino en el aula de clase.

Tal vez sería necesario empezar hablando de reeducación, de la ciudadanía y del docente. Pero no solamente a este hay que reeducarle en el uso de esas nuevas herramientas que, al fin y al cabo, son eso herramientas. Desconocemos en gran parte la tecnología porque no nos han formado en ella. Nacimos analógicos y nos han metido con calzador en un mundo digital. Ya teníamos bastante con la televisión y sus avances: más de un canal, emisiones en color, mando a distancia, frecuencias por cable, pantallas planas, puertos USB,...

Sería más importante que, como docentes, nos formásemos y nos educáramos en valores, en ciudadanía, en humanidad. Educar a los educadores para que puedan educar y formar a los educandos, que son la ciudadanía. La formación, 
desde la familia a la Universidad, quienes lleguen a ella, que tampoco es una condición indispensable para una vida digna, pasando por la calle y la escuela, nos dota de sentido y entidad, nos enriquece y nos conciencia. Esa educación formativa comienza cuando nacemos y termina al morir, pasemos o no por una institución formal, nos acompaña toda la vida, estimulando nuestro crecimiento como personas y nuestra conciencia para jugar nuestro papel en la vida.

Cierto que con la educación no nos hacemos económicamente más ricos, ni políticamente más poderosos, ni más fuertes físicamente. Pero sí, al menos eso deseamos, más humanos, más educados, más ciudadanos(as). En ese sentido, y como parte fundamental del proceso educativo, ¿es la Universidad uno de los problemas de hoy?, ¿o es una posible solución?

El profesor Ken Robinson, en sus estudios sobre la escuela, ha observado que chicas y chicos pierden la creatividad con la edad y que lo que todos ellos tienen en común es que han pasado por un centro educativo (Robinson, 2006). A Herman Hesse la escuela solamente le sirvió para aprender latín, que no es poco, y para decir mentiras y, además, le había destrozado muchas cosas. Aun así decía que la "lectura sin amor, el saber sin respeto, la formación sin corazón" eran "uno de los mayores pecados contra el espíritu" (Hesse, 1981, p. 83).

Creo que la Universidad, pese a todos sus problemas y contradicciones, es una institución necesaria. Porque es menester universalizar la razón y el pensamiento, hacer público el conocimiento y contribuir, desde la educación superior, a la formación de seres humanos en el más amplio sentido del término. La Universidad está expuesta a varios peligros que la "apartan" del lugar esencial que debe ocupar en la sociedad. Por un lado los lobos externos, el capitalismo, el neoliberalismo, las privatizaciones, el mercantilismo; y, por otro, esos caballos de Troya que hacen que se fagocite a sí misma, que su endogamia le nuble el sentido y que su falta de visión le impida ver la realidad en la que estamos.

Si la educación es un acto político que requiere un comportamiento ético, en la Universidad ese comportamiento tiene que ser más visible y contundente. Porque la educación tiene un papel muy destacado en la formación de una ciudadanía crítica y comprometida, y en la construcción de espacios de debate democrático; y es en las instituciones universitarias donde se concentra esa parte de la población que se supone se educa, no solo para tener mejor formación sino una mejor calidad de vida y la posibilidad de mejorar la de los demás.

La formación ética y moral en la Universidad debe transversalizar el pensum académico. Solamente así podemos pretender lograr la educación ciudadana y que ésta contribuya a la integración.

La vida es compleja y la Universidad no puede ser en exceso protectora o abandonar a su suerte a sus estudiantes. Todas y todos tenemos nuestros pensamientos, sentimientos, creencias, intereses, usos y rutinas; tenemos formas distintas de ser y estar en el mundo que se han insertado en nuestro carácter a través de los tres ámbitos educativos básicos: la familia, la calle y la escuela. 
Tal como plantean Pagés y Santisteban para la escuela, deberíamos proponer para la Universidad: que todos los saberes tiendan a que las y los estudiantes se formen como miembros de una ciudadanía "capaz de convivir con los demás $\mathrm{y}$ tomar decisiones sobre su futuro como personas, miembros de una familia y de una comunidad".

Esa debería ser una de las tareas básicas y fundamentales, y añadiría que incluso fundacionales, de la institución universitaria. Su visión y su misión tienen que atender a la formación de una ciudadanía crítica y comprometida, desde el respeto, la comprensión y la solidaridad.

La Universidad es una apuesta política, por eso debería ser pública, accesible y gratuita. Y debe formar una ciudadanía también política. Porque en la vida todo es política, en el sentido de estrategia de actuación de los individuos y los grupos sociales para alcanzar sus objetivos. Debe formar con criterio a una ciudadanía que debe tener un pensamiento crítico que la capacite para trabajar por una sociedad incluyente e integradora. Como decía Zenón de Elea: “dichosa la ciudad donde se admira menos la hermosura de los edificios que las virtudes de sus habitantes".

González Valencia, Posada López y Valencia Calvo plantean que:

Una adecuada formación ciudadana debe ser liberadora y debe lograr en los estudiantes el reconocimiento de los derechos y deberes individuales y colectivos. Por otra parte, la formación de ciudadanos para la participación va más allá de los contenidos, porque esto requiere enseñar a los estudiantes a pensar y reflexionar sobre la vida cotidiana y comprender que el mundo se caracteriza por su diversidad: social, cultural, étnica, religiosa, etc. (En Alba, N., García, F., y Santisteban, A., 2012, p. 126).

Hoy el mundo necesita diálogo intercultural, requiere de un reconocimiento de la alteridad (de la condición de ser otro) que se tiene que dar en las aulas y en los patios universitarios. Antes de tecnificarse en exceso, que no estaría mal pero no debería ser la prioridad, la Universidad debe promover el intercambio, la interacción y el contacto, evitando caer en la virtualidad que termina excluyendo esa comunicación educativa, esa educomunicación que se da en el aula de clase. La Universidad no tiene que olvidar a su ciudadanía nativa y sus culturas para dedicarse a las élites y a proveer el mercado de mano de obra.

Gutiérrez Girardot ya anunciaba, hace 50 años, los síntomas que seguimos padeciendo:

El primero y más grave de todos es la pérdida del sentido del estudio universitario. El Estado y las élites le han arrebatado al estudio su esencia antropológica, es decir, el deseo y la pasión por el conocimiento, y han transformado su propósito social, el anhelo por construir un país más justo, en una mercancía más del 
mercado y, en las actuales circunstancias, hasta en una mercancía deleznable (2011, p. 13).

También Galeano nos advierte cuando dice que:

La escuela del mundo al revés es la más democrática de las instituciones educativas. No exige examen de admisión, no cobra matrícula y gratuitamente dicta sus cursos, a todos y en todas partes, así en la tierra como en el cielo: por algo es hija del sistema que ha conquistado, por primera vez en toda la historia de la humanidad, el poder universal (Galeano, 1999, p. 5).

\section{El mito de las clasificaciones}

A eso contribuye que vivimos condicionados por las clasificaciones, los rankings, los ratings, el share y demás zarandajas impuestas desde el poder económico para categorizar, excluir y medir la competitividad. Cuando lo que realmente nos debería preocupar es la competencia, entendida como capacidad, como la "pericia, aptitud, idoneidad para hacer algo o intervenir en un asunto determinado" (DRAE). Ni la ciudadanía, ni las universidades, ni por supuesto las TIC escapan de esa fiebre. Hoy todo se clasifica, se cataloga y se tipifica agrupando los ítems en torno a lo que el sistema demanda y exige.

Las tres clasificaciones más consultadas mundialmente para universidades son la elaborada por la Universidad china Jiao Tong de Shanghai (SJTU), la que publica la revista británica Times Higher Education (THE, anteriormente THES), y la española Webometrics realizada por el Centro Superior de Investigaciones Científicas (CSIC) y su laboratorio de Cibermetría.

Si consideramos la ciudadanía y sus atributos (responsabilidad, solidaridad y comprensión del otro), y miramos las clasificaciones universitarias, vemos que en ninguno de los criterios aparece rasgo alguno de ciudadanía para hacer la catalogación. Pero tampoco hay medidas tecnológicas. Si repasamos los rankings mencionados más arriba, vemos que no hay criterios humanos o sociales, sino de renombre y de prestigio. Una reputación que, reconociendo su valor científico, tiene más que ver con cualidades de oferta y demanda que de compromiso ciudadano.

De las tres mencionadas, la única que podríamos decir que cuenta con las TIC es la tercera. Webometrics tiene como objetivo "promover la publicación en la web académica, mediante el apoyo a las iniciativas de acceso abierto, con el fin de incrementar significativamente la transferencia de conocimientos científicos y culturales generados por las universidades a toda la sociedad". Centra su ranking en las habilidades que tienen las universidades para publicar en Internet. A partir de métodos cuantitativos, ha elaborado unos indicadores cibermétricos (1), que complementan los tradicionales bibliométricos (2), con los que se puede 
estudiar la actividad científica en la web. Esos criterios van desde el número de publicaciones en revistas internacionales indexadas, hasta las citas en trabajos publicados, pasando por premios internacionales de sus estudiantes y académicos, o contenidos en Internet o artículos en revistas de impacto.

(1) Cibermetría: es la disciplina dedicada a la descripción cuantitativa de los contenidos y procesos de comunicación que se producen en el ciberespacio (Aguillo, 2003, p. 6)

(2) Según Ruiz de Osma, del Departamento de Biblioteconomía y Documentación. Universidad de Granada (España), los indicadores bibliométricos "permiten averiguar el impacto causado por un trabajo científico cualquiera a partir de la literatura científica y tecnológica publicada"; y también "manejar, clasificar y analizar grandes volúmenes de publicaciones científicas".

El Webometrics empezó su andadura en el 2004 y, desde 2006, se edita dos veces al año (enero y julio) recogiendo datos de casi doce mil universidades de todo el mundo. El estudio, que pretende ser un "ranking académico e independiente" no tiene fines de lucro, por lo que se le puede considerar más "riguroso y objetivo" que aquellos otros que tienen detrás algún interés económico, político o comercial.

Tal como señalan en su página web:

El objetivo no es evaluar páginas web, su diseño o usabilidad o la popularidad de su contenido, de acuerdo con el número de visitas o diversidad de visitantes (...), los indicadores web de presencia e impacto pueden ser un mecanismo importante y objetivo para la correcta evaluación, completa y profunda, de las actividades universitarias, de su gestión y gobernanza, de los servicios que ofrecen, de la calidad de su docencia e investigación, y de la relevancia e impacto de los resultados científicos, tecnológicos, culturales o económicos tanto a nivel local como internacional. (www.webometrics.info/es)

En la clasificación que elabora el CSIC las diez primeras universidades en su listado son:

\begin{tabular}{|c|l|c|c|c|c|c|}
\hline Ranking & Universidad & País & $\begin{array}{c}\text { Presencia } \\
\text { puesto }\end{array}$ & $\begin{array}{c}\text { Impacto } \\
\text { puesto }\end{array}$ & $\begin{array}{c}\text { Apertura } \\
\text { puesto }\end{array}$ & $\begin{array}{c}\text { Excelencia } \\
\text { puesto }\end{array}$ \\
\hline 1 & Harvard U. & EE.UU. & 64 & 1 & 19 & 1 \\
\hline 2 & $\begin{array}{l}\text { Massachusetts, } \\
\text { Inst. of Techn. MIT }\end{array}$ & EE.UU. & 7 & 1 & 57 & 13 \\
\hline 3 & Stanford U. & EE.UU. & 11 & 4 & 11 & 2 \\
\hline 4 & $\begin{array}{l}\text { U. California, } \\
\text { Berkeley }\end{array}$ & EE.UU. & 645 & 3 & 18 & 15 \\
\hline
\end{tabular}




\begin{tabular}{|c|l|c|c|c|c|c|}
\hline Ranking & \multicolumn{1}{|c|}{ Universidad } & País & $\begin{array}{c}\text { Presencia } \\
\text { puesto }\end{array}$ & $\begin{array}{c}\text { Impacto } \\
\text { puesto }\end{array}$ & $\begin{array}{c}\text { Apertura } \\
\text { puesto }\end{array}$ & $\begin{array}{c}\text { Excelencia } \\
\text { puesto }\end{array}$ \\
\hline 5 & $\begin{array}{l}\text { U. California, Los } \\
\text { Ángeles (UCLA) }\end{array}$ & EE.UU. & 1008 & 5 & 69 & 3 \\
\hline 6 & U. Washington & EE.UU. & 837 & 6 & 8 & 6 \\
\hline 7 & U. Michigan & EE.UU. & 23 & 11 & 21 & 5 \\
\hline 8 & Cornell U. & EE.UU. & 639 & 7 & 10 & 22 \\
\hline 9 & U. Minnesota & EE.UU. & 909 & 8 & 23 & 20 \\
\hline 10 & U. Pennsylvania & EE.UU. & 13 & 14 & 67 & 9 \\
\hline
\end{tabular}

En cuanto a las universidades de Latinoamérica, las tres primeras son la Universidad de Sao Paulo (USP, Brasil) en el puesto 31, la Autónoma de México (UNAM, México) en el 70, y la Universidad del Estado de Campiñas (Unicamp, Brasil) en el 274. Para que se hagan una idea comparativa con un país "desarrollado", la primera española es la Universidad Complutense de Madrid en el puesto 91. Las tres primeras de Colombia son la Universidad Nacional en el 479, la de los Andes en el 752 y la de Antioquia en el puesto 945.

Esta clasificación hace uso de una proporción 1:1 entre indicadores de actividad, a través de la presencia, la apertura y la excelencia, con base en temas como las publicaciones y contenidos en la web, y los indicadores de impacto, es decir, la visibilidad web medida a partir del número de enlaces externos recibidos.

Por su parte, la THE califica cuatrocientas universidades internacionales en función de factores como la "calidad de la investigación", que puntúa el $60 \%$; la "capacidad de que un graduado obtenga empleo", valorada en un $10 \%$; la "presencia internacional", que contabiliza un $10 \%$, y el "cociente entre estudiantes y académicos" que suma el $20 \%$ restante.

Su ranking correspondiente al periodo 2012-2013, donde muestra que los diez primeros puestos los ocupan universidades del Reino Unido y de los Estados Unidos:

\begin{tabular}{|c|l|c|c|}
\hline Ranking & \multicolumn{1}{|c|}{ Universidad } & País & Puntuación \\
\hline 1 & California, Institute of Technology (Caltech) & EE.UU. & 95.5 \\
\hline 2 & U. Oxford & U.K. & 93.7 \\
\hline 3 & Stanford U. & EE.UU. & 93.7 \\
\hline 4 & Harvard U. & EE.UU. & 93.6 \\
\hline 5 & Massachusetts, Inst. of Techn. MIT & EE.UU. & 93.1 \\
\hline 6 & Princeton U. & EE.UU. & 92.7 \\
\hline 7 & U. Cambridge & U.K. & 92.6 \\
\hline 8 & Imperial College, London & U.K. & 90.6 \\
\hline 9 & U. California, Berkeley & EE.UU. & 90.5 \\
\hline 10 & U. Chicago & EE.UU. & 90.4 \\
\hline
\end{tabular}


La primera Universidad latinoamericana es la de Sao Paulo (Brasil) en el puesto 158 con una puntuación de 50.5. La Universidad de los Andes es la única representante colombiana en la posición 352.

Por lo que se refiere a la Universidad china Jiao Thong de Shanghai, valora quinientas instituciones universitarias basándose en un procedimiento que tiene en cuenta los siguientes factores y porcentajes para realizar su clasificación: el número de premios Nobel o de medallas Fields que se han adjudicado, lo que supone un $10 \%$ de la valoración; o qué enseñan en la institución universitaria, que anota un $20 \%$; la cantidad de investigadores "altamente citados en 21 temas generales", que registra un $20 \%$; el número de artículos aparecidos en las revistas científicas Nature y Science, que puntúa un $20 \%$; los trabajos académicos que aparecen en registros como el Social Science Citation Index o el Science Citation Index, que significa otro $20 \%$, y una puntuación que se calcula dividiendo los anteriores factores entre el número de académicos a tiempo completo que tiene la Universidad, que añade el otro $10 \%$.

En sus datos relativos a 2013, nos revelan que en los primeros diez lugares de la clasificación aparecen ocho universidades de los Estados Unidos de América y dos de Gran Bretaña:

\begin{tabular}{|c|l|c|c|c|}
\hline $\begin{array}{c}\text { Ranking } \\
\text { mundial }\end{array}$ & \multicolumn{1}{|c|}{ Universidad } & País & $\begin{array}{c}\text { Ranking } \\
\text { nacional }\end{array}$ & Puntuación \\
\hline 1 & Harvard U. & EE.UU. & 1 & 100 \\
\hline 2 & Stanford U. & EE.UU. & 2 & 72.6 \\
\hline 3 & U. California, Berkeley & EE.UU. & 3 & 71.3 \\
\hline 4 & $\begin{array}{l}\text { Massachusetts, Inst. of Techn. } \\
\text { MIT }\end{array}$ & EE.UU. & 4 & 71.1 \\
\hline 5 & U. Cambridge & U.K. & 1 & 69.6 \\
\hline 6 & $\begin{array}{l}\text { California, Institute of Technology } \\
\text { (Caltech) }\end{array}$ & EE.UU. & 5 & 62.9 \\
\hline 7 & Princeton U. & EE.UU. & 6 & 61.9 \\
\hline 8 & Columbia U. & EE.UU. & 7 & 59.8 \\
\hline 9 & U. Chicago & EE.UU. & 8 & 57.1 \\
\hline 10 & U. Oxford & U.K. & 2 & 55.9 \\
\hline
\end{tabular}

La Universidad brasileña de Sao Paulo vuelve a ser la primera latinoamericana en aparecer clasificada ocupando el puesto 146. En este listado no aparece ninguna Universidad colombiana.

\section{Propuestas}

Y se preguntarán ustedes para qué les cuento todo esto de las universidades. Pues, en primer lugar, por motivos de conocimiento general, y en segundo 
lugar, no menos importante, porque me parece interesante conocer estos datos para hacer una valoración, subjetiva por supuesto, de esa relación Universidad, ciudadanía y TIC.

De las clasificaciones mencionadas, ¿podríamos extrapolar que los países que aparecen en los primeros diez lugares (en todas ellas EE.UU. y Gran Bretaña) tienen mejores universidades?, pues tal vez sí, aunque con base en criterios que no cuentan con las personas ni con la situación social, y sí con la economía que permite a la mayoría de las "grandes" universidades invertir ingentes cantidades de dinero en sus aulas.

Siguiendo los criterios que estudian esas ordenaciones y viendo las cifras que reportan con ellos, ¿estaríamos en capacidad de afirmar que las TIC juegan un papel destacado en el ranking?, yo diría que no. Salvo la lista de Webometrics, que se realiza a partir de datos basados en Internet y en las páginas web de las instituciones, no hay criterios tecnológicos "puros" a la hora de evaluar.

Y por último, observando todos los datos que nos presentan las tres listas más consultadas para hacer evaluación de la supuesta calidad académica universitaria, ¿nos dicen algo acerca de si esos países son mejores o cuentan con mejores ciudadanas y ciudadanos?, pues afirmaría rotundamente que no.

Entonces, ¿por qué esa pelea por aparecer en los listados de clasificación de las universidades? Sencillamente porque da imagen frente al poder económico y pareciera que la institución, sus docentes y sus egresados son más "fiables". Craso error, porque lo importante somos las personas y no los productos.

En ese orden clasificatorio, tampoco encontramos que los premios científicos más prestigiosos del mundo, los Premios Nobel, tengan uno al ciudadano ético, o a la ciudadana responsable, al colectivo solidario y participativo, a la vecina comunicadora o al paisano escuchante. Además, qué fiabilidad pueden tener unas distinciones que recompensan con el premio de la Paz a personajes como el norteamericano Kissinger o los israelíes Rabin y Peres pero no reconocieron a Gandhi.

Pero sí hay unos galardones que premian a organizaciones y personas que destacan por su trabajo a favor de un futuro mejor para el planeta y sus habitantes. Son los llamados Premios Nobel Alternativos, los Right Livelihood, que desde su creación en 1980 por el filántropo sueco-alemán Jakob von Uexkull, han entregado sus laureles a más de ciento cincuenta instituciones y personalidades de 64 países.

Sus reconocimientos destacan a quienes hayan defendido los derechos humanos, la justicia social, la paz, la infancia, el medioambiente o la ciencia, o hayan luchado contra el hambre o las armas, y son premios abiertos a cualquier ciudadano o ciudadana del mundo. Cualquier persona puede presentar su candidato.

Los premios al "correcto modo de vida" pretenden, en palabras de su creador, "ayudar al Norte para que alcance una sabiduría a la altura de su ciencia y al 
Sur para que desarrolle una ciencia a la altura de su sabiduría milenaria" (von Uexkull, http://www.rightlivelihood.org/summary_spanish.html)

Entre quienes han "cumplido" con el antiguo principio de "dedicarse a una ocupación honrada, respetuosa de los seres humanos y del entorno natural" y ser personas que se responsabilizan "de sus actos y se limitan a consumir una porción justa de los recursos del planeta”, destacan el activista, educador y músico venezolano José Antonio Abre y el teólogo y profesor brasileño Leonardo Boff; el Festival Internacional de Poesía de Medellín (Colombia); el Grupo de Agricultura Orgánica de Cuba; el Movimiento de los Sin Tierra de Brasil; el político y economista chileno Manfred Max-Neef y el arquitecto y cofundador del Foro Social Mundial Chico Whitaker.

El filósofo y maestro colombiano Gutiérrez Girardot dice que “el estudio es una pasión, no una profesión, una aventura y un riesgo, no una carrera, un fuego, no un acto burocrático, el desafío de la libertad" (Gutiérrez, 2011, p. 50).

El planteamiento final de esta reflexión es que debemos luchar contra esas universidades mercantiles que aprovechan un parqueadero para dictar clase, y lo que dictan es lo que dicta el mercado. En Colombia parece que Colciencias, ente superior que "democratiza" la investigación, la docencia y a docentes e investigadores, sigue los pasos que manda el capital transnacional y presenta exigencias de cara a una clasificación y medición de investigadores y grupos que cumplen más con el capital que con la ciudadanía. ¿Qué mide y con qué criterios? Los países mal llamados en vías de desarrollo, no tienen que seguir el desarrollo de los "desarrollados", no se deben conformar con ser clientes del sistema económico mundial.

Las universidades tienen que tener dentro al país y a sus paisanos. Desde la Universidad se ha de repensar la ciudadanía, se ha de levantar la voz por la justicia social y si para todo ello es necesario hacer uso de las tecnologías, pues adelante; pero como herramientas para lograr un objetivo social, no como condición indispensable para promover y mantener la exclusión y que se amplíe, no solo la brecha digital, sino, y más importante, la social.

Señala G. Girardot que:

El saber no se satisface con lo logrado, el saber consiste también en ponerse permanentemente en tela de juicio. El saber que no se pone en tela de juicio se convierte en dogma, y el dogma, entiéndase por tal opinión o artículo de fe, petrifica el saber, lo esteriliza (2011, p. 35).

Y reclama:

Una Universidad cuyo objetivo sea no solo el de la formación profesional sino el de la creación y producción de saberes y de nuevos planteamientos. Esto último contribuye a su vez a una mejor formación profesional, pues el profesional que durante su 
formación participa de la creación y de la producción de saber, transmite esa dinámica a la sociedad misma y cumple así con una de las tareas del universitario, esto es, la de servir al mejoramiento y adelanto de la sociedad" (Ibid., p. 36).

Universidad y TIC pueden hacer mucho por una ética colectiva en la ciudadanía. La cuestión es cómo se reconozca y cómo actúe aquella y qué uso se haga de estas.

\section{Como conclusión}

Podríamos plantear, al igual que proponían los teóricos de la dependencia para superarla, que la Universidad, para tener más sociedad dentro y ser más activa fuera:

- Atienda a la sociedad civil, de la que como institución forma parte y de la que no debería aislarse.

- Presente propuestas académicas e investigativas para el fortalecimiento de la participación social, no solo de docentes y estudiantes sino también de la ciudadanía.

- Colabore con organizaciones sociales en busca de una mayor presencia social de aquellas a través de trabajos y proyectos conjuntos incluyentes.

- Valore los elementos culturales e ideológicos de la diversidad, que conviven en la sociedad y en el interior de la Universidad, como motores de la movilización social. Esta es necesaria para que la Universidad y la sociedad no se anquilosen.

- Respalde la movilización social para promover el desarrollo, buscar la transformación y trabajar por la cohesión social y la integración.

Todas estas acciones y sus valores implícitos se suponen que están incluidos en la misión y la visión de las universidades. Los múltiples choques de civilizaciones que nos plantean los agoreros de la historia, sin ser tales, conllevan un peligro para la integración y la igualdad. La Universidad debe englobar esas diferencias para convertir los choques en diálogos interculturales. Reconocer nuestras debilidades nos permite transformarnos y conformar humanidad.

Según el profesor Pérez Mantilla, "la Universidad ha estado siempre en crisis. Su inadecuación al saber y a su tiempo la hicieron temblar en la Edad Media, en el Renacimiento, en la Francia de Napoleón y la Alemania de Humboldt". Pero la idea de Universidad, su labor universalista, tiene que perdurar si realmente forma país y forma ciudadanía.

La labor cultural y formativa de la institución universitaria tiene que servir de contrapeso al utilitarismo de la sociedad. Para ello tiene que ser autónoma, no dependiente y menos aún de las tecnologías, y suponer, como decía Humboldt, "una isla de libertad y saber". 
En este mundo altamente tecnificado y globalizado, para ciertas cosas, la ciencia y el conocimiento son poderes, forman parte del proceso productivo. Y la Universidad, en donde se concentran la ciencia, el conocimiento, la investigación y la producción académica, si se encuentra sometida a la mercantilización y al industrialismo pierde su carácter universalista y queda convertida en productora de mano de obra especializada para el mercado.

Ya no es cultural ni crítica, ya no forma ciudadanía sino obreros baratos y consumidores. Queda al servicio de la economía y del capital. La Universidad no debe ser eso. Tiene que promover la formación y dotar de conocimiento crítico a una ciudadanía crítica. Porque con el conocimiento y el criterio puede contribuir a la integración social. Las obras, cuando son culturales y sociales, no se acaban nunca. El conocimiento no tiene fin y la Universidad no debería tenerlo si en verdad se dedicara a ello.

La educación es un proceso de comprensión de la realidad para poderla transformar. Tenemos que tomar conciencia de quiénes somos, ciudadanos y docentes, y de dónde estamos, en la Universidad, para que, a través de la duda y del pensamiento crítico, podamos problematizar, motivar y buscar caminos para un desarrollo más humano y más humanista. Freire decía que "ante lo nuevo, el hombre de conciencia crítica, no rechaza lo viejo por viejo; ni acepta lo nuevo por serlo, sino en la medida en que son válidos" (Freire, 1981, p. 14).

No permitamos que el mundo al revés nos imponga esa escuela. No consintamos que nos impongan esas tecnologías que nos someten al poder y al mercado. $Y$ si esa es "su" escuela, luchemos, desde todos los ámbitos educativos, la familia, la calle, la academia, y con todas las herramientas, incluidas las TIC, por otra bien distinta. Una verdadera escuela, o una contraescuela.

\section{Referencias}

Brecht, B. (1999). Poemas y canciones. Madrid: Alianza editorial.

Derrida, J. (1997). Las pupilas de la Universidad. El principio de razón y la idea de la Universidad. En Cómo no hablar y otros textos. Barcelona: Proyecto A. Edición digital en castellano. Disponible en http://www.jacquesderrida. com.ar/textos/universidad.htm

Freire, P. (1981). Educación y cambio. Río de Janeiro: Paz y Tierra.

Galeano, E. (1999). Patas arriba. La escuela del mundo al revés. Madrid: Siglo XXI editores.

Giner, S. (s.f.). Ciudadanía pública y democracia participativa. Disponible en http:// www.alcoberro.info/V1/republica6.htm 
González, V., Posada, L., y Valencia, C. (2012). La enseñanza para la participación en el currículo de Ciencias Sociales en la educación obligatoria en Colombia. En Alba, N., García, F., y Santisteban, A. Educar para la participación ciudadana en la enseñanza de las ciencias sociales, (p. 126).

Gutiérrez, R. (2011). La encrucijada universitaria. Medellín: Asoprudea.

Hesse, H. (1981). Lecturas para minutos. Madrid: Alianza editorial.

Martín, J. (2013). Una agenda de comunicación para este país, conferencia inaugural del semestre 2013-2 de la Maestría en Comunicación, Desarrollo y Cambio Social de la Universidad Santo Tomás.

Robinson, K. (2006). Las escuelas matan la creatividad. En TED, Ideas worth spreading. Disponible en http://www.ted.com/talks/lang/es/ken_robinson_says_schools_kill_creativity.html

\section{Webgrafía}

Centro Superior de Investigaciones Científicas (CSIC): http://www.webometrics. info/es/

Premios Nobel Alternativos: http://www.rightlivelihood.org/summary_spanish. html

Times Higher Education (THE): http://www.timeshighereducation.co.uk/ world-university-rankings/2012-13/world-ranking

Universidad Jiao Tong de Shanghai (SJTU) http://www.shanghairanking.com/ ARWU2012.html 